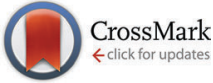

Cite this: Phys. Chem. Chem. Phys., 2015, 17, 13829

Received 24th March 2015, Accepted 20th April 2015

DOI: $10.1039 / c 5 c p 01711 d$

www.rsc.org/pccp

\title{
Generating hydrated electrons through photoredox catalysis with 9-anthrolate $\dagger$
}

\author{
Christoph Kerzig and Martin Goez*
}

\begin{abstract}
Hydrated electrons are among the strongest reductants known. Adding the ascorbate dianion as a sacrificial donor turns the photoionization of 9-anthrolate in water into a catalytic cycle for their in situ production with near-UV light $(355 \mathrm{~nm})$. The photoionization step is exclusively biphotonic and occurs via the first excited singlet state of the catalyst. Neither triplet formation nor any photochemical side reactions interfere. The ionization by-product, the anthroxy radical, is inert towards the ascorbate monoanion but is rapidly reduced by the dianion, thereby recovering the starting catalyst. A sufficient amount of the sacrificial donor makes that reduction quantitative and leads to a sustainable generation of hydrated electrons, as is evidenced by electron yields greatly surpassing the catalyst concentration. Control experiments established that the superincrease is indeed due to the catalyst regeneration and not to an ionization of other species involved in the reaction.
\end{abstract}

\section{Introduction}

The interest in photoredox catalysis as a synthetic tool has been growing exponentially during the last few years. ${ }^{1-11}$ This method exploits that an excited state is both a better electron acceptor and a better electron donor than the ground state; it thus takes advantage of the photon energy to prepare a stronger oxidant or a reductant as an intermediate that activates a substrate through a (direct or mediated) photoinduced electron transfer; sacrificial donors or acceptors, although less favourable from the point of view of atom economy, ${ }^{8,9}$ provide a very flexible way of regenerating the photocatalyst.

It seems to be natural to extend this idea to less reactive substrates by utilizing two photons to afford a "super oxidant" or a "super reductant" in situ. The archetypal example of the latter is the solvated or hydrated electron $\mathbf{e}_{\mathbf{a q}}{ }^{\bullet-}\left(E^{\circ}=2.77 \mathrm{~V} v\right.$ s. normal hydrogen electrode NHE, comparable to activated alkali metals), ${ }^{12}$ which has already been shown to be capable of reductively detoxifying chloro- ${ }^{13,14}$ and fluoroorganics ${ }^{15}$ as well as directly reducing molecular nitrogen ${ }^{16}$ and carbon dioxide. ${ }^{17}$ Generating $\mathbf{e}_{\mathbf{a q}}{ }^{\bullet-}$ with a single photon necessitates energy-rich light (UV-C in all the cited examples), which is strongly absorbed by many substrates. However, the two-photon approach to $\mathbf{e}_{\mathbf{a q}}{ }^{\bullet-}$ elegantly circumvents that potential problem by shifting the required wavelengths to the $\mathrm{UV}-\mathrm{A}^{18}$ or even the visible. ${ }^{19}$ As a photoreleased "super reductant", $\mathbf{e}_{\mathbf{a q}}{ }^{\bullet-}$ should have one intrinsic

Martin-Luther-Universität Halle-Wittenberg, Institut für Chemie, Kurt-Mothes-Str. 2, D-06120 Halle (Saale), Germany. E-mail: martin.goez@chemie.uni-halle.de $\dagger$ Electronic supplementary information (ESI) available. See DOI: 10.1039/ c5cp01711d advantage over an excited radical anion, which was successfully employed for that purpose recently, ${ }^{20}$ namely a much longer natural life, microseconds (compare, Fig. 4 and 5) against nanoseconds or shorter.

Depending on whether the photocatalyst is an electron acceptor or an electron donor, two basic catalytic cycles that produce $\mathbf{e}_{\mathbf{a q}}{ }^{\bullet-}$ by consuming two photons and a sacrificial donor are conceivable. As opposed to the numerous examples we have reported for the former (Scheme 1a) ${ }^{18,19,21-25}$ none are known for the latter. In this work, we present a detailed mechanistic investigation of the first photocatalytic system according to Scheme $1 b$, where the catalyst is recovered by a thermal reaction with a sacrificial donor after the photoionization.

The anions of hydroxy-substituted aromatic compounds appear to be particularly well suited for the purpose because they are very good electron donors, their ionic nature increases

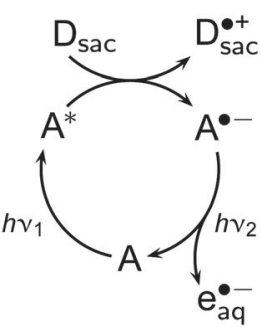

(a)

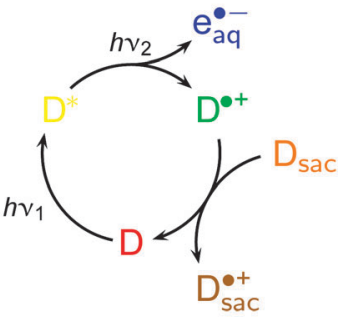

(b)
Scheme 1 Turning the photoionization of (a) an electron acceptor A and (b) an electron donor $D$ into a catalytic cycle by a sacrificial donor $D_{\text {sac }}$. The colour code for all the species in (b) is used throughout this study. For further explanation, see the text. 
the solubility in the aqueous medium, and-as the main advantage over their protonated parent compounds-they cannot undergo homolytic cleavage to give hydrogen atoms as a competing reaction to photoionization. ${ }^{26}$ Increasing the size of the conjugated system brings a twofold benefit, namely, a shift of the absorption towards the red and a reduction of the ionization energy: phenolate can only be ionized in the UV-C owing to its absorption properties, ${ }^{26,27}$ whereas 2-naphtholate is already (biphotonically) ionizable at $337 \mathrm{~nm},{ }^{28}$ via its first excited singlet state $S_{1}$. For the present work, we have selected the higher homologue 9-anthrolate, which we excite into $S_{2}$ with $355 \mathrm{~nm}$; the 1,8-dihydroxy derivative of that anion is thought to be the active form of a popular antipsoriasis agent. $^{29}$

Our experimental method is two-pulse-two-colour laser flash photolysis. To minimize interpretational uncertainties, we monitor all species occurring in the reaction mechanism, Scheme $1 \mathrm{~b}$.

\section{Results and discussion}

\subsection{Relevant ground- and excited-state properties of the photocatalyst}

In aqueous medium, the enol 9-anthrol enjoys merely a fleeting existence owing to two fast equlilibria, one for its deprotonation to give the anthrolate $\mathbf{A O L}^{-}\left(\mathrm{p} K_{\mathrm{a}} \approx 7.8\right)$, the other for its tautomerization to give the ketone $10 H$-anthr-9-one AON $(K \approx$ 150). ${ }^{30}$ As summarized in Scheme 2 , this results in an apparent single equilibrium between the last two species with a $\mathrm{p} K_{\mathrm{a}}^{\prime}$ value of 10, which facilitated choosing conditions such as to allow the photoionization of $\mathbf{A O L}^{-}$to be studied without interference from other forms of that compound, namely, sufficiently high $\mathrm{pH}$ (mostly above 12) combined with laser excitation at $355 \mathrm{~nm}$, where the molar absorption coefficient of $\mathbf{A O L}^{-}$dwarfs that of AON by almost two orders of magnitude. Quantum-mechanical calculations indicate (compare, Section S1 of the ESI $\dagger$ ) that our photocatalyst $\mathbf{A O L}{ }^{-}$is best described as a carbanion.

As opposed to $\mathbf{A O N}, \mathbf{A O L}^{-}$is extremely susceptible to oxidation by molecular oxygen, the end product being 9,10-anthraquinone. ${ }^{31}$ To circumvent this in preparing our solutions, we exploited the

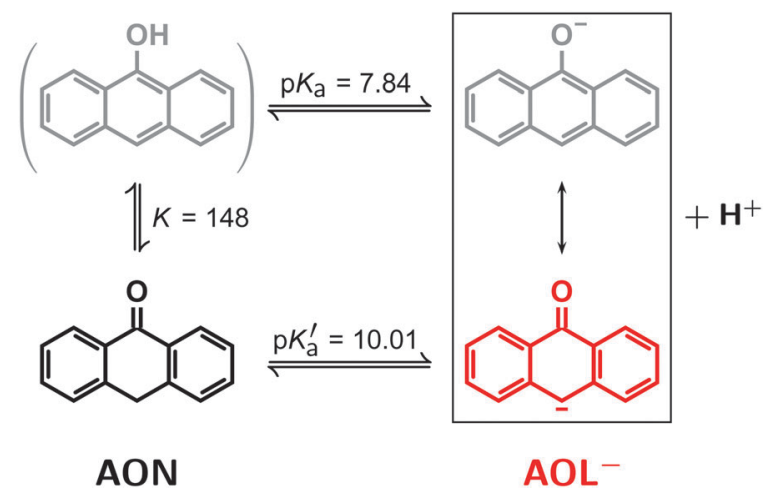

Scheme 2 Different forms of the photocatalyst in its ground state. Owing to the displayed fast equilibria ${ }^{30}$ only the ketone $A O N$ and the anthrolate $\mathrm{AOL}^{-}$are relevant. For further information, see the text. above-mentioned fast equilibria by adding the required amount (typically, one percent of the final volume) of a freshly made and deoxygenated millimolar solution of AON in iso-propanol to a deoxygenated aqueous solution at the desired $\mathrm{pH}$ using syringe techniques. Even though iso-propanol is a very good hydrogen donor, its presence does not interfere with the reactions of Scheme $1 \mathrm{~b}$, as evidenced by control experiments (see below). Spectrophotometry on the final solutions before and after each series of experiments served to determine the actual $\mathbf{A O L}^{-}$ concentration and stability, and in particular to ascertain the absence of quinone.

Fig. 1 contains the absorption and luminescence spectra of $\mathrm{AOL}^{-}$.

In the $\mathrm{pH}$ range $(10.5 \leq \mathrm{pH} \leq 13)$ and concentration range $\left(5 \mu \mathrm{M} \leq\left[\mathbf{A O L}^{-}\right] \leq 40 \mu \mathrm{M}\right)$ used in this work, we did not detect any deviations from the Beer-Lambert law nor changes in the spectral shape. The $S_{1}$ and $S_{2}$ excited states of AOL ${ }^{-}$correspond to the featureless ${ }^{1} \mathrm{~L}_{\mathrm{a}}$ and to the vibrationally structured ${ }^{1} \mathrm{~L}_{\mathrm{b}}$ band, ${ }^{32}$ at $433 \mathrm{~nm}$ and 359/376 $\mathrm{nm}$ in our medium. The higherenergy vibrational feature of the latter transition almost perfectly matches our excitation wavelength $(355 \mathrm{~nm})$, and the lower-energy subpeak $\left(\varepsilon_{376}=9900 \mathrm{M}^{-1} \mathrm{~cm}^{-1}\right)$ allows a convenient monitoring of the $\mathbf{A O L}{ }^{-}$ground-state concentration. A contamination by oxidation can be detected with good sensitivity in the region of low absorbance at around $330 \mathrm{~nm}$, where anthraquinone absorbs strongly (spectrum not shown).

The fluorescence spectrum of $\mathbf{A O L}^{-}$(maximum at $525 \mathrm{~nm}$ ) exhibits a mirror-image relationship with the ${ }^{1} \mathrm{~L}_{\mathrm{a}}$ absorption band. The curve crossing at $487 \mathrm{~nm}$ puts the energy of the $0-0$ transition at $2.55 \mathrm{eV}$. Because this is considerably less than the formation energy of $\mathbf{e}_{\mathbf{a q}}{ }^{\bullet-}$, the $S_{1}$ state obviously cannot autoionize. Neither $S_{1}$ nor $S_{2}$ are quenched by iso-propanol, as experiments with different alcohol concentrations showed.

We determined the fluorescence quantum yield $\varphi_{\text {lum }}$ against fluorescein as the standard, and at low absorbance $(A \leq 0.1)$ to

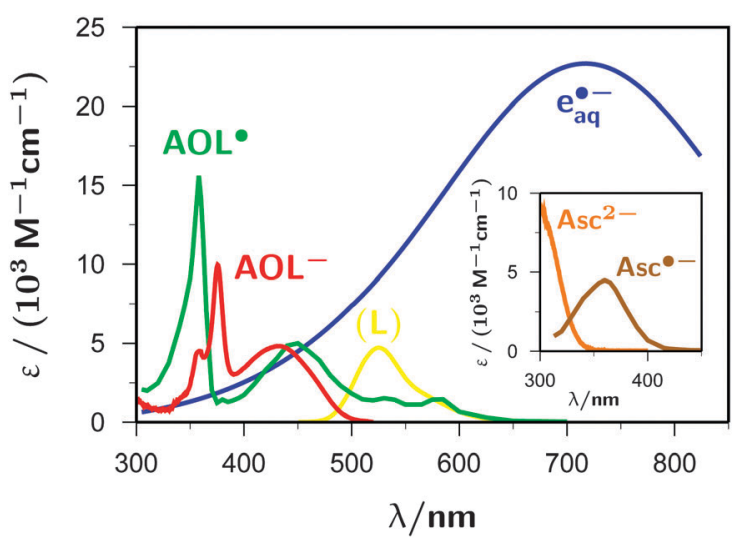

Fig. 1 Calibrated absorption spectra-except for the curve labelled L, which is the uncalibrated luminescence spectrum of the anthrolate $\mathrm{S}_{1}$ - of all species relevant for this work, with the same colour coding as in Scheme 1b; the assignment is given by the labels at the curves. The inset has exactly the same horizontal and vertical scale as the main plot, and merely serves to avoid overcrowding in the region below $450 \mathrm{~nm}$. For further explanation, see the text. 
avoid inner filter effects. These measurements gave a wavelengthindependent value of $0.073 \pm 5 \%$ between 355 and $420 \mathrm{~nm}$, which indicates that the ${ }^{1} \mathrm{~L}_{\mathrm{b}}$ state $\left(\mathrm{S}_{2}\right)$ also does not autoionize to a significant degree. As a corollary, $\mathbf{e}_{\mathbf{a q}}{ }^{\bullet-}$ formation should not have a pronounced monophotonic component.

To estimate the excited-state life, we carried out quenching experiments with $\mathrm{I}^{-}$. We found no evidence for the formation of ground-state complexes, and we obtained a linear Stern-Volmer plot with a Stern-Volmer constant of $15.8 \mathrm{M}^{-1}$. Modifying the accepted value for a diffusion controlled reaction between uncharged species in water $\left(6.5 \times 10^{9} \mathrm{M}^{-1} \mathrm{~s}^{-1}\right)^{33}$ by the Debye formula ${ }^{34}$ with an encounter distance of $5.6 \AA$ (the sum of the vander-Waals radius of $\mathrm{I}^{-}$and the geometric mean of the molecular radii of $\mathbf{A O L}{ }^{-}$in the direction of the three principal axes; for the latter, we added the van-der-Waals radii of the terminal atoms to the quantum-mechanically computed distances $\mathrm{O}-\mathrm{H}_{10}$ and $\mathrm{H}_{2}-$ $\mathrm{H}_{7}$, and took the separation of adjacent layers in graphite as the third component), ${ }^{35}$ we obtained a quenching rate constant of $3.2 \times 10^{9} \mathrm{M}^{-1} \mathrm{~s}^{-1}$, so a singlet lifetime $\tau_{\mathrm{S}}$ of $4.9 \mathrm{~ns}$. The latter value is practically identical to the duration $\tau_{\mathrm{L}}$ of our laser pulses (5.0 ns).

A sensitization experiment served as the test for a potential participation of the triplet state in the photochemistry of $\mathbf{A O L}^{-}$ at $355 \mathrm{~nm}$. The sensitizer naphthalene possesses a long-lived (17 $\mu \mathrm{s}$ as measured in our medium) and efficiently formed $\mathrm{T}_{1}$, which lies even slightly above the $\mathrm{S}_{1}$ of $\mathbf{A} \mathbf{O L}^{-}$, so is expected to undergo rapid triplet-triplet energy transfer to the $\mathbf{A O \mathbf { L } ^ { - }}$ ground state; ${ }^{33}$ its generation is feasible with $308 \mathrm{~nm}$, i.e., a wavelength within the low-absorptivity window of $\mathbf{A O L}^{-}$in the UV. By employing a naphthalene concentration of $400 \mu \mathrm{M}$, which necessitated increasing the iso-propanol admixture to $10 \%$ to overcome the limited solubility of the arene in water, an $\mathbf{A O L}^{-}$ concentration of $10 \mu \mathrm{M}$, similar to that in most experiments of this work, and a very low energy of the $308 \mathrm{~nm}$ laser pulse we were able to suppress the photoionizations of naphthalene and $\mathbf{A O L}^{-}$such as to be nondetectable while still producing a sufficient amount of the naphthalene $T_{1}$ and an observable degree of quenching. This resulted in two new bands centered at 330 and $475 \mathrm{~nm}$, the rise time of which was equal to the decay time (15 $\mu \mathrm{s}$ in the two-component system) of the naphthalene triplet at $390 \mathrm{~nm} .{ }^{36}$ Absent were the absorptions of the anthroxy radical $\mathbf{A O L}^{\bullet}$ (Fig. 1) and, in particular, those of the naphthalene radical anion around $700 \mathrm{~nm},{ }^{18}$ so an electron transfer from the naphthalene triplet is ruled out, and the quenching mechanism can only be energy transfer. On these grounds, we must assign these bands to the $\mathrm{T}_{1}$ state of $\mathbf{A O \mathbf { L } ^ { - }}$ or to a secondary product of that state formed through rapid quenching, e.g., by the isopropanol; in any case, their occurrence is indicative of an involvement of the $\mathbf{A O L}^{-}$triplet. Because these signals-which fall into spectral regions free from other peaks (compare, Fig. 1) and would thus be difficult to overlook-did not arise in all other experiments of this work, we conclude that the triplet state of $\mathbf{A O L}^{-}$does not play any role in our system.

\subsection{Photoionization step}

Irradiation of $\mathbf{A O L}^{-}$with a sufficiently intense $355 \mathrm{~nm}$ pulse leads to photoionization, as is evidenced by the typical signature of the hydrated electron $\mathbf{e}_{\mathbf{a q}}{ }^{\bullet-}$, a very intense and broad band centered slightly above $700 \mathrm{~nm} .{ }^{12}$ For the calibrated spectrum of Fig. 1, we prepared $\mathbf{e}_{\mathbf{a q}}{ }^{\bullet-}$ independently, i.e., directly in our reaction medium but without $\mathbf{A O L}{ }^{-}$, by two-photon ionization of water with $266 \mathrm{~nm},{ }^{37}$ and set the molar absorption coefficient at maximum (718 $\mathrm{nm}$ under our conditions) to the recently redetermined value of $22700 \mathrm{M}^{-1} \mathrm{~cm}^{-1}$. ${ }^{38}$ Our iso-propanol admixture does not react with $\mathbf{e}_{\mathbf{a q}}{ }^{\bullet-}$. $^{12}$ The signals of $\mathbf{e}_{\mathbf{a q}}{ }^{\bullet-}$ and the other transients are conveniently separated by exploiting that only $\mathbf{e}_{\mathbf{a q}}{ }^{\bullet-}$ absorbs above $700 \mathrm{~nm}$ in our system; hence, we simply weighted an absorption trace in that region with the appropriate ratio of molar absorption coefficients and subtracted it from the trace at a shorter wavelength of interest.

In this way, we obtained the spectrum (Fig. 1) of the anthroxy radical $\mathbf{A O L}^{\bullet}$, the necessary by-product of the photoionization of $\mathbf{A O L}^{-}$. The primary separation result is an uncalibrated difference spectrum of $\mathbf{A O L}{ }^{\bullet}$ and $\mathbf{A O L}^{-}$, which is characterized by a positive maximum at $360 \mathrm{~nm}$ and a negative minimum of almost equal magnitude at $375 \mathrm{~nm}$. The former indicates a strong absorption of $\mathbf{A O L}^{\bullet}$ at our excitation wavelength, so the question arises whether AOL $^{\bullet}$ might itself be photoionizable (to give a nonradical cation). This issue-which is crucial not only for a unique spectral decomposition but also for a correct analysis of the intensity dependences-can be resolved by an absorbance-difference diagram, ${ }^{39}$ with the modification of varying the reaction progress by varying the laser intensity: the absorbance changes at these two key wavelengths and at, e.g., $824 \mathrm{~nm}$ (a wavelength that allows a very sensitive monitoring of $\mathbf{e}_{\mathbf{a q}}{ }^{\bullet-}$, for instrumental reasons explained in the Experimental section) are found to be strictly proportional to one another over the accessible turnover range; hence, $\mathbf{A O L}^{-}, \mathbf{A O L}^{\bullet}$, and $\mathbf{e}_{\mathbf{a q}}{ }^{\bullet-}$ are connected by a single reaction step, and there is no possibility of a photoreaction consuming $\mathbf{A O L}^{\bullet}$.

Fig. 2 reproduces this absorbance-difference diagram in a slightly modified form, using concentrations instead of absorptions. This recalculation is based on our observation (see below) that the addition of a sufficiently high amount of the ascorbate dianion completely restores the starting concentration of $\mathbf{A O L}^{-}$in the dark period after the laser flash, which establishes that the photoionization is not accompanied by any other photochemical reaction, so not only affords identical amounts of $\mathbf{A O L}^{\bullet}$ and $\mathbf{e}_{\mathbf{a q}}{ }^{\bullet-}$ but also consumes the very same amount of $\mathbf{A O L}^{-}$. This stoichiometric relationship allows a precise calibration of the molar absorption coefficient of $\mathbf{A O L}^{\bullet}$ against that of $\mathbf{e}_{\mathbf{a q}}{ }^{\bullet-}$; the obtained value for $\varepsilon\left(\mathbf{A O L}^{\bullet}\right)$ at the $358 \mathrm{~nm}$ maximum is $15600 \mathrm{M}^{-1} \mathrm{~cm}^{-1}$. Because both the signal to be calibrated and the reference absorption is derived from the same experiment, all uncertainties of the optical path length are eliminated.

As is explained above, our preparation method necessitated an iso-propanol content of the solutions of typically $1 \%$, i.e., $0.13 \mathrm{M}$. To test whether a reaction of $\mathbf{A O L}^{\bullet}$ with this good hydrogen donor needs to be taken into account, we increased the iso-propanol concentration by a factor of twenty in a control experiment. This should accelerate a hydrogen abstraction by the same factor, so should shorten the life of $\mathbf{A O L}^{\bullet}$ if that 


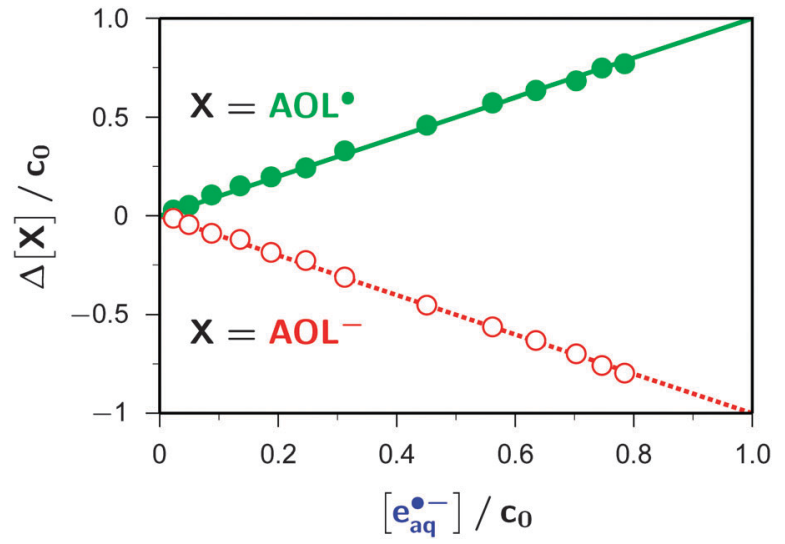

Fig. 2 Concentration changes $\Delta[X]$ as a function of the electron concentration $\left[\mathbf{e}_{\mathbf{a q}}{ }^{--}\right]$, all taken relative to the starting anthrolate concentration $c_{0}$ $(11.5 \mu \mathrm{M})$; red open circles, for $\mathbf{A O L}^{-}$; green filled circles, for $\mathbf{A O L}$. The broken red and solid green lines have (negative and positive) unit slope and pass through the origin. Experimental $\mathrm{pH}, 12.3$; each triple of concentration changes recorded at constant intensity of a $355 \mathrm{~nm}$ laser flash; maximum laser intensity, $315 \mathrm{~mJ} \mathrm{~cm}^{-2}$. Wavelengths for the concentration determinations were $824 \mathrm{~nm}\left(\mathbf{e}_{\mathbf{a q}^{-}}{ }^{-}\right), 375 \mathrm{~nm}$ (bleaching of $\mathrm{AOL}^{-}$) and $360 \mathrm{~nm}\left(\mathbf{A O L}^{\bullet}\right)$. For further explanation, see the text.

reaction played a role; instead, we observed a doubling of the $\mathbf{A O L}^{\bullet}$ life, which can be quantitatively rationalized by the effect of the higher solution viscosity ${ }^{40}$ on the diffusion-controlled dimerization of $\mathbf{A O L}^{\bullet}$ and recombination with $\mathbf{e}_{\mathbf{a q}}{ }^{\bullet-}$. The same control experiment also ruled out an influence of the isopropanol admixture on the photoionization: the initial absorbance directly after the laser pulse changed by as little as $5 \%$, which can be traced back to a slight solvatochromic shift.

Fig. 3 displays intensity dependencies not only for the $\mathbf{e}_{\mathbf{a q}}{ }^{\bullet-}$ concentration but also for the luminescence (compare, Fig. 1) originating from the first excited state of $\mathbf{A O L}^{-}$; owing to the short life of that state, the total (i.e., integrated) luminescence

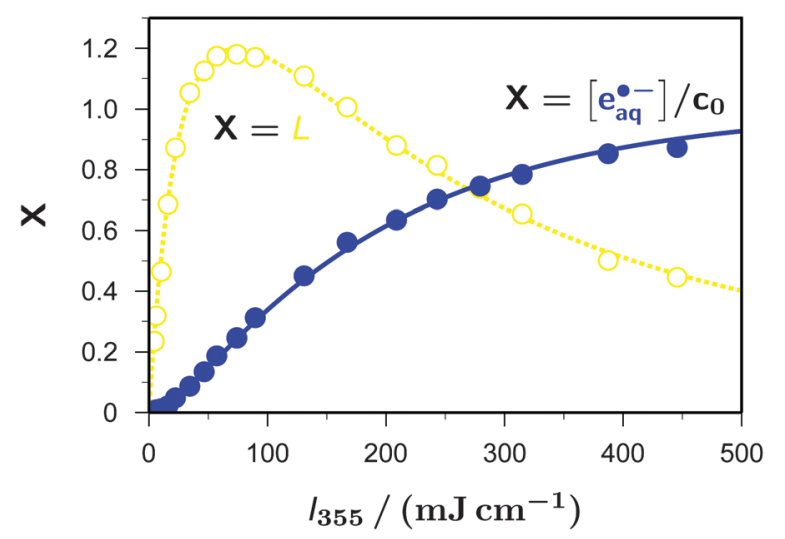

Fig. 3 Dependence of the $\mathbf{e}_{\mathbf{a q}}{ }^{\bullet-}$ concentration relative to the starting catalyst concentration $c_{0}$ (blue filled circles and solid line) and of the integrated luminescence $L$ (yellow open circles and dashed line) on the laser intensity $I_{355}$; experimental parameters, $c_{0}=11.5 \mu \mathrm{M}, \mathrm{pH} 12.3$, $L$ observed at $520 \mathrm{~nm}$. The lines were calculated by a simultaneous fit of eqn (S6) and (S8) of the ESI, $\dagger$ with fixed parameter $\varphi_{\text {mono }}=0$. For further explanation, see the main text and Section S2 of the ESI. $\dagger$ was used. It is obvious that more reliable and detailed information about a potentially complex photoreaction mechanism can be obtained by observing more than one species.

These intensity dependencies can be fitted perfectly with the kinetic model explained in Section S2 of the ESI. $\dagger$ Simultaneous fits to both data sets require adjusting only two intensityproportional photokinetic parameters (one for the primary excitation, and one for the ionization of the $S_{1}$ excited state by a second photon) and two constants (the quantum yield $\varphi_{\text {mono }}$ of spontaneous electron ejection from $\mathrm{S}_{1}$, and a scaling factor for the luminescence because our laser flash photolysis setup is not capable of measuring absolute luminescence).

The already mentioned wavelength-independence of $\varphi_{\text {lum }}$ up to wavelengths where the energy balance of electron ejection from $S_{1}$ becomes thermodynamically unfavourable militates against a significant monophotonic contribution to electron formation. Yet, those features of the electron yield as a function of the laser intensity that are indicative of a biphotonic ionization, namely, an upward curvature in the low-intensity range and a deviation from zero of the intercept when the curve is back-extrapolated linearly, ${ }^{41}$ are almost absent with that system (compare, Fig. 3). The origin of such a phenomenon is known to be an unfavourable combination of molar absorption coefficients and quantum yields; ${ }^{41}$ as an aggravation, in the relevant intensity region the electron signal is smallest, so the sensitivity is lowest, which further hampers the analysis.

On the following grounds, the simultaneous monitoring of the electron concentration and the luminescence appears to be better suited to the task. Because luminescence emission and monophotonic electron formation originate from the same intermediate through the same type of process (i.e., with light-independent rate each), the amount of $\mathbf{e}_{\mathbf{a q}}{ }^{\bullet-}$ from the monophotonic pathway must be strictly proportional to the total luminescence, with the constant of proportionality being $\varphi_{\text {mono }} / \varphi_{\text {lum }}$ (compare, eqn (S6) and (S8) of the ESI $\dagger$ ). As a corollary, a high degree of similarity of the intensity dependencies of $L$ and of the electron concentration would thus indicate a predominance of the monophotonic ionization pathway. The method further has the advantage that the observables are analyzed over the whole intensity range, not only within the region of the intrinsically worst signal-to-noise ratio.

Treating the complete parameter set as adjustable gave a best-fit value of only 0.007 for $\varphi_{\text {mono }}$ and no better representation of the experimental data than a fit with $\varphi_{\text {mono }}$ set to zero. In accordance with the principle of Occam's razor and the preceding reasoning based on the wavelength-independence of $\varphi_{\text {lum }}$, we take this to mean that there is no monophotonic ionization in our system. Despite the need of absorbing a second photon during the short life of the $\mathrm{S}_{1}$ excited state, near-quantitative ionization of the catalyst is nevertheless attainable with a single laser pulse, as Fig. 3 shows.

\subsection{Control experiments on the sacrificial donor}

To turn this photoionization into a catalytic cycle according to Scheme $1 \mathrm{~b}$ by regenerating the electron source $\mathbf{A O L}^{-}$from its radical $\mathbf{A O L}^{\bullet}$, we need to add a sacrificial donor that must be a 
strong, water-soluble reductant and should absorb as weakly as possible at the ionization wavelength, $355 \mathrm{~nm}$. All these requirements are met by the well-known antioxidant ascorbic acid (vitamin C). Its fully protonated form $\left(\mathrm{p} K_{\mathrm{a} 1}=4.25\right)^{42}$ plays no role at all in our basic medium; only its monoanion HAsc ${ }^{-}$ $\left(\mathrm{p} K_{\mathrm{a} 2}=11.79,{ }^{42} E^{\circ}\left(\mathbf{H A s c}^{\bullet} / \mathbf{H A s c}^{-}\right)=0.300 \mathrm{~V} v s\right.$. normal hydrogen electrode $\mathrm{NHE}^{43}$ ) and-dominating at our typical $\mathrm{pH}$ well above 12-its even more strongly reducing dianion $\mathbf{A s c}^{2-}\left(E^{\circ}\left(\mathbf{A s c}^{\bullet-}\right)\right.$ Asc $^{2-}$ ) $=0.015$ V vs. NHE) ${ }^{43}$ are relevant.

At $355 \mathrm{~nm}, \mathbf{H A s c}^{-}$is not photoionized, but $\mathbf{A s c}^{2-}$ produces a small amount of $\mathbf{e}_{\mathrm{aq}}{ }^{\bullet-}$ despite its minute molar absorption coefficient (see, Fig. 1). We corrected for that effect in all the experiments on mixed $\mathbf{A O L}{ }^{-}-\mathbf{A s c}^{2-}$ systems by subtracting the $\mathbf{e}_{\mathbf{a q}}{ }^{\bullet-}$ and $\mathbf{A s c}^{\bullet-}$ signals obtained in experiments without $\mathbf{A O L}{ }^{-}$ but otherwise identical; in the worst case, this correction amounted to less than $10 \%$.

The control experiment displayed in Fig. 4 addresses the question of whether $\mathbf{A s c}^{\bullet-}$ is photoionizable with $355 \mathrm{~nm}$, a wavelength almost coinciding with its absorption maximum in the near UV. To that end, we first prepared $\mathbf{A s c}^{\bullet-}$ by $308 \mathrm{~nm}$ photoionization of $\mathbf{A s c}^{2-}$, which gave the spectrum of the radical shown in Fig. 1 as an additional benefit; by an absorbance-difference diagram similar to Fig. 2, we ascertained the absence of a $308 \mathrm{~nm}$ photoreaction of $\mathbf{A s c}^{\bullet-}$, so established the stoichiometric relationship between $\left[\mathbf{e}_{\mathbf{a q}}{ }^{\bullet-}\right]$ and $\left[\mathbf{A s c}^{\bullet-}\right]$ necessary for precisely calibrating the $\mathbf{A s c}^{\bullet-}$ molar absorption coefficient at maximum $\left(\varepsilon_{360}=4500 \mathrm{M}^{-1} \mathrm{~cm}^{-1}\right)$. Having produced $\mathbf{A s c}^{\bullet-}$ and $\mathbf{e}_{\mathbf{a q}}{ }^{\bullet-}$ by a $308 \mathrm{~nm}$ pulse, we then applied an intense $355 \mathrm{~nm}$ pulse after a short delay. As is evident from Fig. 4, that second pulse has no effect on the slow decay of the $\mathbf{A s c}^{\bullet-}$ concentration and does not produce any new electrons. Hence, Asc $^{\bullet-}$ is photoionizable neither with $308 \mathrm{~nm}$ nor with $355 \mathrm{~nm}$.

Such experiments also provide clear evidence that $\mathbf{A s c}^{\bullet-}$ is very stable in our medium except for its recombination with $\mathbf{e}_{\mathbf{a q}}{ }^{\bullet-}$ : the $\mathbf{A s c}^{\bullet-}$ concentration exhibits a small initial decay,

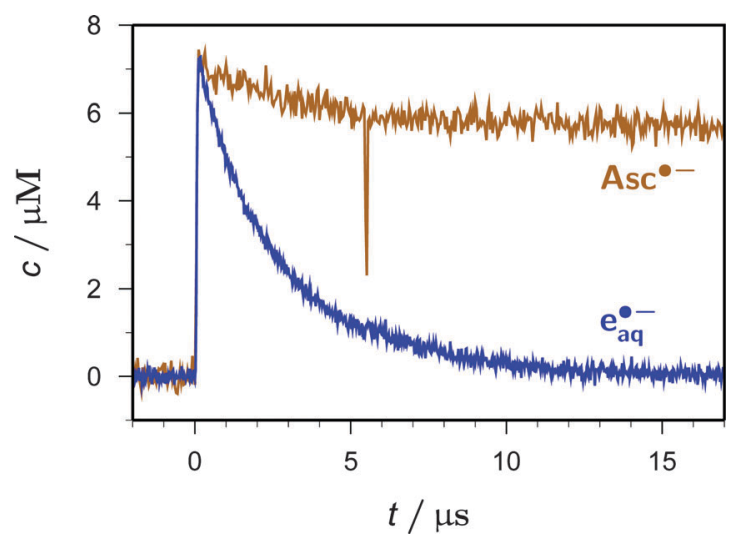

Fig. 4 Two-pulse experiment (first pulse at $t=0,308 \mathrm{~nm}, 280 \mathrm{~mJ} \mathrm{~cm}{ }^{-2}$; second pulse at $t=5.5 \mu \mathrm{s}, 355 \mathrm{~nm}, 212 \mathrm{~mJ} \mathrm{~cm}^{-2}$ ) on a $50 \mu \mathrm{M}$ solution of $\mathbf{A s c}^{2-}$ at pH 12.4 showing the time dependence of the $\mathbf{A s c}^{\mathbf{C}^{-}}$(brown) and $\mathbf{e}_{\mathbf{a q}}{ }^{\cdot-}$ (blue) concentrations. The negative spike of the Asc ${ }^{\cdot-}$ curve at the moment of the second pulse is an artifact caused by insufficient stray-light suppression because the excitation and observation wavelengths differed by $5 \mathrm{~nm}$ only. For further explanation, see the text. by some $15 \%$ in Fig. 4 , which comes to a complete standstill after $\mathbf{e}_{\mathbf{a q}}{ }^{\bullet-}$ has vanished. A quantitative comparison of the electron decay in the absence of $\mathbf{A s c}^{\bullet-}$ (i.e., when we generated the same amount of $\mathbf{e}_{\mathbf{a q}}{ }^{\bullet-}$ from water with $266 \mathrm{~nm}$ at identical $\mathrm{pH}$ ) and in its presence merely gave the difference expected from that recombination; hence, scavenging of $\mathbf{e}_{\mathbf{a q}}{ }^{\bullet-}$ by $\mathbf{A s c}^{2-}$ plays no role under our conditions. Lastly, at the concentrations used in this work $\mathbf{A s c}^{2-}$ does not react with the $\mathrm{S}_{1}$ excited state of $\mathbf{A O L}^{-}$, as follows from the lack of a luminescence quenching.

\subsection{Regeneration step}

Fig. 5a and b juxtapose the concentration traces for $\mathbf{A O L}^{-}$and $\mathbf{e}_{\mathbf{a q}}{ }^{\bullet-}$ in two-pulse experiments $(355 \mathrm{~nm} / 355 \mathrm{~nm})$ that were identical twins except for the addition of $\mathbf{A s c}^{\mathbf{2}-}$ in the second case. The first pulse produces the same concentration of $\mathbf{e}_{\mathbf{a q}}{ }^{\bullet-}$ in both experiments, about $85 \%$ of the starting catalyst concentration, which is accompanied by a decrease of the $\mathbf{A O L}^{-}$ concentration to about $15 \%$ of its inital value.

In the absence of $\mathbf{A s c}^{\mathbf{2}-}$, the recombination of $\mathbf{e}_{\mathbf{a q}}{ }^{\mathbf{}-}$ with $\mathbf{A O L}^{\bullet}$ inefficiently (and, of course, undesirably because it removes the species of interest) regains about the same amount of $\mathbf{A O L}^{-}$during the interpulse delay (Fig. 5a). In contrast, when $\mathbf{A s c}^{\mathbf{2}-}$ has been added, this sacrificial donor largely suppresses that recombination through competition, thus even slightly lengthening the life of $\mathbf{e}_{\mathbf{a q}}{ }^{\bullet-}$ stemming from the first pulse, and instead regenerates $\mathbf{A O L}{ }^{-}$almost completely (Fig. 5b).

The second pulse finally acts on the $\mathbf{A O L}^{-}$molecules available at that point of time, so-in keeping with their concentration ratio-affords more than three times the amount of $\mathbf{e}_{\mathbf{a q}}{ }^{--}$in the solution containing $\mathbf{A s c}^{2-}$. In the examples, the total amount of $\mathbf{e}_{\mathbf{a q}}{ }^{\bullet-}$ extracted from the system by two laser flashes is thus found to surpass the starting catalyst concentration only slightly in the absence of $\mathbf{A s c}^{\mathbf{2}-}$ (and that excess is merely due to the described recombination artifact) but by more than $60 \%$ in the presence of the sacrificial donor $\mathbf{A s c}^{2-}$. Furthermore, it is clearly seen from the second post-flash period in Fig. 5b that this sequence of ionization and regeneration could be repeated a substantial number of times until the system were exhausted.

Without ascorbate, and after $\mathbf{e}_{\mathbf{a q}}{ }^{\bullet-}$ has died down, the further fate of $\mathbf{A O L}^{\bullet}$ is dimerization to give one or several products absorbing below $370 \mathrm{~nm}$. While these absorbances have only a negligible influence on the quantitative monitoring of $\mathbf{A O L}^{-}$at $375 \mathrm{~nm}$, as can be seen in Fig. 5a (the minute apparent rise of the $\mathbf{A O L}^{-}$trace in the interval between 10 and $20 \mu \mathrm{s}$ ), they do interfere with the concentration determination of $\mathbf{A O L}^{\bullet}$ at our usual wavelength, $360 \mathrm{~nm}$. We, therefore, observed the $\mathbf{A O L}^{\bullet}$ decay at above $500 \mathrm{~nm}$ where no other species besides $\mathbf{e}_{\mathbf{a q}}{ }^{\bullet-}$ absorbs (Fig. 1). In $\mathrm{N}_{2} \mathrm{O}$-saturated solution, where $\mathbf{e}_{\mathbf{a q}}{ }^{\bullet-}$ is quantitatively removed within the duration of our laser pulses through a scavenging cascade, ultimately yielding dimethyl ketyl radical anions in our medium, ${ }^{12}$ we found a clean second-order decay of $\mathbf{A O L}^{\bullet}$ over an $80 \mu$ s time interval with a rate constant $k_{\mathrm{d}}$ of $2 \times 10^{9} \mathrm{M}^{-1} \mathrm{~s}^{-1}$. We obtained the same value when we repeated the experiment in Ar-saturated solution and analyzed only the part 
(a)

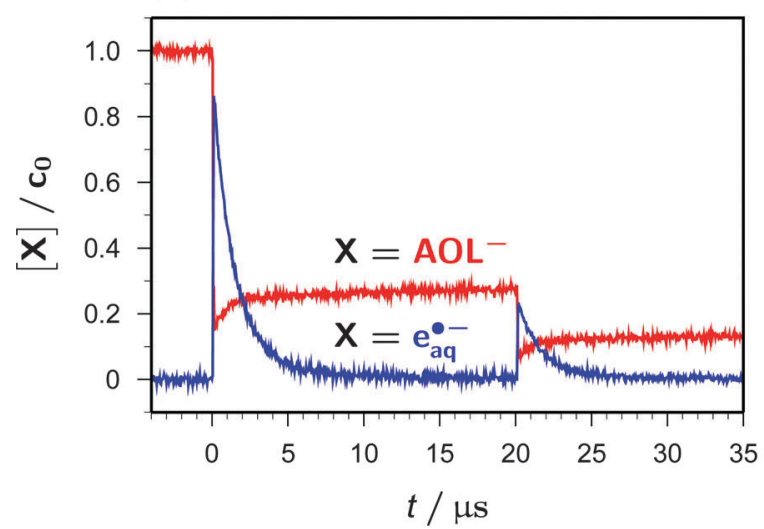

(b)

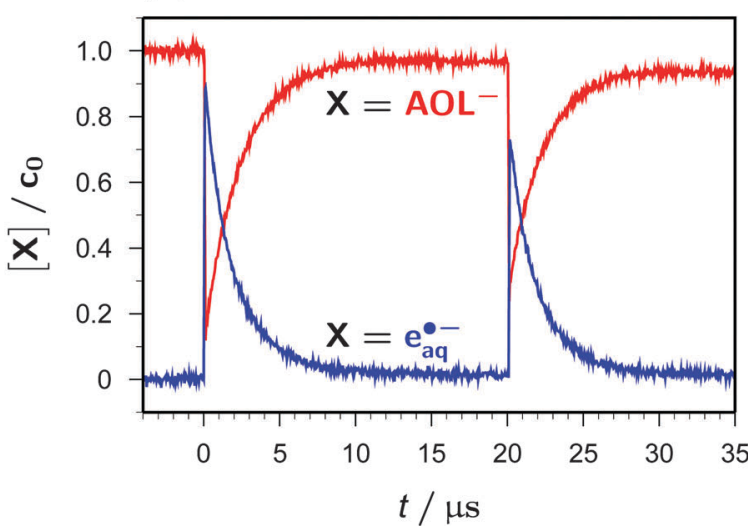

Fig. 5 Two-pulse (both $355 \mathrm{~nm}$ ) laser flash photolysis on an $11.9 \mu \mathrm{M}$ solution of $\mathbf{A O L}^{-}$at $\mathrm{pH}$ 12.4; interpulse delay, $20 \mu \mathrm{s}$; first pulse, $437 \mathrm{~mJ} \mathrm{~cm}^{-2}$; second pulse, $308 \mathrm{~mJ} \mathrm{~cm}^{-2}$. Shown are the concentration traces of $\mathbf{e}_{\mathrm{aq}}{ }^{-{ }^{-}}$(blue) and $\mathbf{A O L}^{-}$(red) scaled to the starting catalyst concentration $c_{0}$. Graph (a), without $\mathrm{Asc}^{2-}$; graph (b), with $0.6 \mathrm{mM} \mathrm{Asc}^{2-}$ added. For further explanation, see the text.

of the trace after $\mathbf{e}_{\mathbf{a q}}{ }^{\bullet-}$ had disappeared, so the thermodynamically feasible secondary production of $\mathbf{A O L}^{\bullet}$ by the attack of the dimethyl ketyl radical anions on residual $\mathbf{A O L}^{-}$must be insignificant.

To determine whether only the dianion $\mathbf{A s c}^{2-}$ is capable of regenerating $\mathbf{A O L}^{-}$from $\mathbf{A O L}^{\bullet}$, or also the monoanion $\mathbf{H A s c}^{-}$, we used the $\mathrm{pH}$ to vary the concentration ratio between HAsc and $\mathbf{A s c}^{2-}$. Fig. 6 shows the outcome of two such experiments, at $\mathrm{pH} 10.8$ and $\mathrm{pH}$ 12.7.

By adjusting the weight-in concentrations of AON according to the protonation equilibria of anthrol (see the above discussion and explanation of the sample preparation method), we ensured equal concentrations of $\mathbf{A O L}^{-}$in both solutions. At the

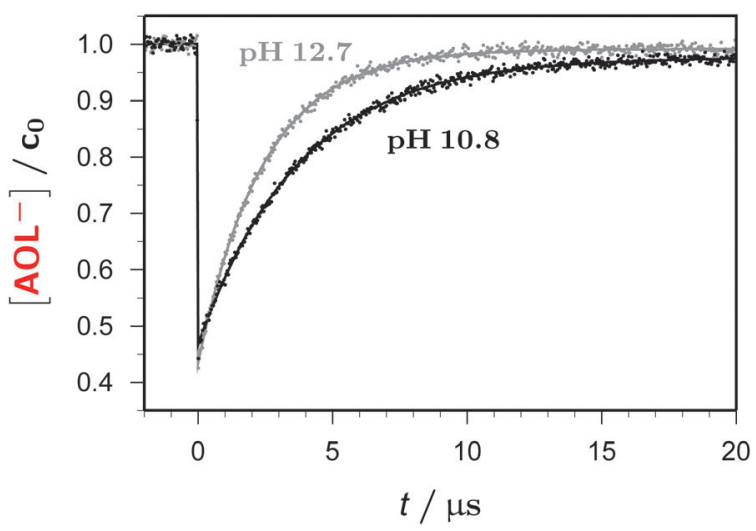

Fig. 6 Laser flash photolysis $\left(355 \mathrm{~nm}, 179 \mathrm{~mJ} \mathrm{~cm}^{-2}\right.$ at time $\left.t=0\right)$ on two solutions containing $13 \mu \mathrm{M} \mathrm{AOL}^{-}$as the actual species concentration plus ascorbate at different $\mathrm{pH}$. The dots represent the measured concentration traces of $\mathrm{AOL}^{-}$normalized to the starting concentration $c_{0}$; the solid curves are best fits of $r_{\infty}-\left(r_{\infty}-r_{0}\right) \exp \left[-k_{r} t\right]$ to each data set after the flash. Black, pH 10.8; weight-in concentration of AON, $15.0 \mu \mathrm{M}$; weight-in concentration of ascorbate, $4.26 \mathrm{mM}$ corresponding to $3.86 \mathrm{mM} \mathrm{HAsc}^{-}$ and $0.395 \mathrm{mM} \mathrm{Asc}^{2-}$; best-fit parameters, $r_{\infty}=0.98, r_{0}=0.47, k_{\mathrm{r}}=2.67 \times$ $10^{5} \mathrm{~s}^{-1}$. Gray, $\mathrm{pH} 12.7$; weight-in concentration of $\mathrm{AON}, 13.0 \mu \mathrm{M}$; weight-in concentration of ascorbate, $0.678 \mathrm{mM}$ corresponding to $74.2 \mu \mathrm{M} \mathrm{HAsc}$ and $0.604 \mathrm{mM} \mathrm{Asc}^{2-}$; best-fit parameters, $r_{\infty}=0.99, r_{0}=0.43, k_{\mathrm{r}}=4.14 \times$ $10^{5} \mathrm{~s}^{-1}$. For further explanation, see the text. lower $\mathrm{pH}$, where the $\mathbf{A O N}$ concentration is about $15 \%$ of the $\mathbf{A O L}^{-}$concentration, we searched for additional transients but found none; hence, neither the triplet nor the radical anion is formed under our conditions.

The integrated rate law for the regeneration of $\mathbf{A O L}{ }^{-}$through a first-order reduction of $\mathbf{A O L}^{\bullet}$ by $\mathbf{A s c}^{2-}$ (rate constant $k_{\mathrm{r}}$ ) competing with a second-order dimerization of $\mathbf{A O L}^{\bullet}$ (rate constant $k_{\mathrm{d}}$ ) can be derived in closed form. Numerically, however, the resulting expression is virtually indistinguishable from the much simpler form $r_{\infty}-\left(r_{\infty}-r_{0}\right) \exp \left[-k_{\mathrm{r}} t\right]$ under the conditions of Fig. $5 \mathrm{~b}$ and 6 , where the dimerization is only a small perturbation. The simpler form is thus to be preferred, in particular, because the additionally competing regeneration of $\mathbf{A O L}^{-}$by the recombination of $\mathbf{A O L}^{\bullet}$ with $\mathbf{e}_{\mathbf{a q}}{ }^{--}$had to be neglected even in the more complete solution. The parameters $r_{0}$ and $r_{\infty}$ are the relative concentrations of $\mathbf{A O L}{ }^{-}$immediately after and at long times after the laser flash; the limiting value $r_{\infty}$ can be related to the kinetic parameters and the starting catalyst concentration $c_{0}$ through the more compete solution,

$$
r_{\infty}=r_{0}+\frac{k_{\mathrm{r}}}{2 k_{\mathrm{d}} c_{0}} \ln \left[1+\frac{2 k_{\mathrm{d}}\left(1-r_{0}\right) c_{0}}{k_{\mathrm{r}}}\right]
$$

but when calculated in this way slightly underestimates the actual value because of the neglect of the recombination of $\mathbf{A O L}^{\bullet}$ with $\mathbf{e}_{\mathrm{aq}}{ }^{\bullet-}$.

The ratio $\left[\mathbf{H A s c}^{-}\right]:\left[\mathbf{A s c}^{2-}\right]$ changes by a factor of 80 between the $\mathrm{pH}$ values of the two experiments, from $9.8: 1$ at $\mathrm{pH} 10.8$ to $0.12: 1$ at $\mathrm{pH}$ 12.7. When we chose appropriate weight-in concentrations of ascorbate to keep the dianion concentrations, hence the rates of $\mathbf{A O L}{ }^{-}$regeneration by $\mathbf{A s c}{ }^{2-}$, within a narrow corridor of $1: 1.53$, we measured the same ratio of reaction rates $(1: 1.55$; the minute deviation is clearly due to experimental uncertainty because otherwise the monoanion reaction would have to have a negative reaction rate).

We, therefore, conclude that within the detection limits of our measurements HAsc $^{-}$does not reduce $\mathbf{A O L}^{\bullet}$ whereas $\mathbf{A s c}^{\mathbf{2}-}$ does; from the best-fit rates given in the caption of Fig. 6, 
the bimolecular rate constant $k$ of the latter reaction is calculated to be $6.8 \times 10^{8} \mathrm{M}^{-1} \mathrm{~s}^{-1}$. Identifying the ability to react with the thermodynamic feasibility, in other words with a more positive potential of the couple $\mathbf{A O L}{ }^{\bullet} / \mathbf{A O L}^{-}$compared to the potential of the pertaining ascorbate-radical/ascorbate couple, $E^{\circ}\left(\mathbf{A O L}^{\bullet} / \mathbf{A O L}^{-}\right)$is thus seen to fall into the range from $0.015 \mathrm{~V}$ to $0.300 \mathrm{~V} v s$. NHE.

The two issues limiting the maximum turnover numbers of the catalytic cycle displayed in Scheme $1 \mathrm{~b}$ are chemical processes competing with the photoionization step or with the regeneration step. Complete restoration of the starting catalyst by the sacrificial donor naturally implies the absence of both problems. An effective $\mathbf{A s c}^{2-}$ concentration of $0.6 \mathrm{mM}$ already suffices to recover $\mathbf{A O L}^{-}$to within $3 \%$ of its initial concentration when the post-flash concentration of $\mathrm{AOL}^{\bullet}$ is $10^{-5} \mathrm{M}$ (Fig. 5b), and to within $1 \%$ of $c_{0}$ when that radical concentration is about halved (Fig. 6, pH 12.7); when the ratio $\left[\mathbf{A s c}^{2-}\right]:\left[\mathbf{A O L}^{\bullet}\right]$ is increased still further, no deviations from a complete recovery can be detected. This trend in the limiting values is perfectly reproduced by eqn (1). These findings rule out a catalyst consumption through photochemical processes other than the photoionization, because the associated catalyst loss could not be undone by $\mathbf{A s c}^{2-}$, and identify radical-radical coupling of $\mathbf{A O L}^{\bullet}$ as the only side reaction competing with the regeneration of of $\mathbf{A O L}^{-}$.

The very low molar absorption coefficient of $\mathbf{A s c}^{2-}$ at $355 \mathrm{~nm}$ allows a substantial concentration increase over $0.6 \mathrm{mM}$ before inner filter effects start to interfere with electron ejection from our photoredox catalyst $\mathbf{A O L}{ }^{-}$. This increase would result in a concomitantly larger amount of $\mathbf{e}_{\mathbf{a q}}{ }^{\bullet-}$ formed by photoionization of our sacrificial donor $\mathbf{A s c}^{\mathbf{2}-}$ itself, an effect entirely unwanted in the mechanistic investigations of this work but entirely welcome in potential applications of our system as an efficient electron source.

\section{Conclusions}

In this work, we have shown and thoroughly investigated a novel mechanism of generating hydrated electrons $\mathbf{e}_{\mathbf{a q}}{ }^{\bullet-}$ through photoredox catalysis, namely, by combining the nearUV (355 nm) ionization of 9-anthrolate with a subsequent thermal reconversion of the resulting 9-anthroxy radical into the starting form of the catalyst using the ascorbate dianion as a sacrificial donor. The photoionization step proved to be efficient enough to produce near-quantitative turnover of the catalyst with a single pulse from a commercial laser, and is entirely free from chemical side reactions, thus allowing a complete regeneration after the pulse. As an obvious asset of such a catalytic cycle for preparing the "super reductant" $\mathbf{e}_{\mathbf{a q}}{ }^{\bullet-}$, only the cheap sacrificial donor is expended whereas the molecule actually liberating the electron is not, so the latter can be tailor-made for the purpose with little regard to cost. While we have not yet performed a test for applications, an interference of the intermediates, products, or by-products formed during the reductive degradation of, e.g., halogenated organic compounds with this catalytic cycle is very unlikely because its only ingredients, anthrolate and ascorbate, are not even attacked by the "super reductant" $\mathbf{e}_{\mathbf{a q}}{ }^{\mathbf{}}$ - and the sacrificial donor ascorbate is a classical free-radical scavenger. Compared to the already known acceptor-based cycle of Scheme 1a, which invariably consumes two photons per electron, the donor-based cycle investigated in this work (Scheme 1b) can equally accommodate a monophotonic ionization of the catalyst, and thus use the photons more efficiently.

\section{Experimental section}

Laser flash photolysis was carried out using a setup described elsewhere. ${ }^{18}$ For each experiment, two of the following three lasers and wavelengths can be combined as desired with freely chosen delay between the pulses: Lambda Physik LPX-210i, $308 \mathrm{~nm}$ with pulse width > $50 \mathrm{~ns}$; continuum Surelite-III and Surelite EX, both $266 \mathrm{~nm}$ or $355 \mathrm{~nm}$ with pulse widths of $5 \mathrm{~ns}$. To provide homogeneous excitation, the beams are individually collimated and sent through the same volume element of a suprasil cell, and the absorptions of the solutions at the laser wavelengths are kept below 0.02 . Transient absorption and luminescence are measured at right angle to the excitation. A syringe-driven flow-through system ensures that each trace is recorded on fresh solution.

We monitored $\mathbf{e}_{\mathbf{a q}}{ }^{\bullet-}$ not at its absorbance maximum but much further in the red, at $824 \mathrm{~nm}$, where the xenon lamp serving as our light source displays a very strong emission spike. This superincrease of the light flux considerably improves the sensitivity, and the $\mathbf{e}_{\mathbf{a q}}{ }^{\bullet-}$ absorption at $824 \mathrm{~nm}$ is still very high; as a beneficial side effect, there are no residual absorptions of other species at that wavelength (see, the spectra in Fig. 1).

AON ( $>99 \%$, Merck) was sublimated several times until no anthraquinone content was detectable. All other chemicals were obtained in the highest available purity and used as received (sodium ascorbate, 99\%, Alfa Aesar; iso-propanol, $>99.7 \%$, Sigma Aldrich; sodium hydroxide for adjusting the pH, 99\%, Grüssing; potassium iodide, puriss., VEB Laborchemie Apolda; fluorescein, >99.5\%, Fluka; naphthalene, 99.6\%, Alfa Aesar). The solvent was ultrapure Millipor MilliQ water (specific resistance, $18.2 \mathrm{M} \Omega \mathrm{cm}$ ); for deoxygenating the solutions, we used argon 5.0 (Linde) or $\mathrm{N}_{2} \mathrm{O} 5.0$ (Air Liquide).

Steady-state absorption and fluorescence spectra were measured using a Shimadzu UV-2102 and a Perkin-Elmer LS 50B spectrometer.

\section{Acknowledgements}

Financial support from the Chemical Industry Funds (PhD scholarship) and the SFi Halle (young researchers award) to C.K. are gratefully acknowledged.

\section{References}

1 K. Zeitler, Angew. Chem., Int. Ed., 2009, 48, 9785-9789.

2 J. M. R. Narayanam and C. R. J. Stephenson, Chem. Soc. Rev., 2011, 40, 102-113. 
3 J. W. Tucker and C. R. J. Stephenson, J. Org. Chem., 2012, 77, 1617-1622.

4 J. Xuan and W.-J. Xiao, Angew. Chem., Int. Ed., 2012, 51, 6828-6838.

5 Chemical Photocatalysis, ed. B. König, DeGruyter, Berlin, 2013.

6 C. K. Prier, D. A. Rankic and D. W. C. MacMillan, Chem. Rev., 2013, 113, 5322-5363.

7 D. Ravelli, M. Fagnoni and A. Albini, Chem. Soc. Rev., 2013, 42, 97-113.

8 M. Reckenthäler and A. G. Griesbeck, Adv. Synth. Catal., 2013, 355, 2727-2744.

9 Y.-Q. Zou, J.-R. Chen and W.-J. Xiao, Angew. Chem., Int. Ed., 2013, 52, 11701-11703.

10 D. P. Hari and B. König, Chem. Commun., 2014, 50, 6688-6699.

11 D. A. Nicewicz and T. M. Nguyen, ACS Catal., 2014, 4, 355-360.

12 J. W. T. Spinks and R. J. Woods, An Introduction to Radiation Chemistry, Wiley, New York, 2nd edn, 1976.

13 X. Li, J. Ma, G. Liu, J. Fang, S. Yue, Y. Guan, L. Chen and X. Liu, Environ. Sci. Technol., 2012, 46, 7342-7349.

14 X. Liu, S. Yoon, B. Batchelor and A. Abdel-Wahab, Sci. Total Environ., 2013, 454-455, 578-583.

15 Z. Song, H. Tang, N. Wang and L. Zhu, J. Hazard. Mater., 2013, 262, 332-338.

16 D. Zhu, L. H. Zhang, R. E. Ruther and R. J. Hamers, Nat. Mater., 2013, 12, 836-841.

17 L. Zhang, D. Zhu, G. M. Nathanson and R. J. Hamers, Angew. Chem., Int. Ed., 2014, 53, 9746-9750.

18 C. Kerzig and M. Goez, Phys. Chem. Chem. Phys., 2014, 16, 25342-25349.

19 M. Goez, C. Kerzig and R. Naumann, Angew. Chem., Int. Ed., 2014, 53, 9914-9916.

20 I. Ghosh, T. Ghosh, J. I. Bardagi and B. König, Science, 2014, 346, 725-728.

21 V. Zubarev and M. Goez, Angew. Chem., Int. Ed. Engl., 1997, 36, 2664-2666.

22 M. Goez, V. Zubarev and G. Eckert, J. Am. Chem. Soc., 1998, 120, 5347-5348.
23 M. Goez and V. Zubarev, Chem. Phys., 2004, 307, 15-26.

24 M. Goez and B. H. M. Hussein, Phys. Chem. Chem. Phys., 2004, 6, 5490-5497.

25 M. Goez and C. Kerzig, Angew. Chem., Int. Ed., 2012, 51, 12606-12608.

26 R. Hermann, G. R. Mahalaxmi, T. Jochum, S. Naumov and O. Brede, J. Phys. Chem. A, 2002, 106, 2379-2389.

27 X. Chen, D. S. Larsen and S. E. Bradforth, J. Phys. Chem. A, 2011, 115, 3807-3819.

28 U. Lachish, M. Ottolenghi and G. Stein, Chem. Phys. Lett., 1977, 48, 402-406.

29 P. J. Hayden and C. F. Chignell, Chem. Res. Toxicol., 1993, 6, 231-237.

30 B. Freiermuth, B. Hellrung, S. Peterli, M.-F. Schultz, D. Wintgens and J. Wirz, Helv. Chim. Acta, 2001, 84, 3796-3809.

31 Y. Ogata, Y. Kosugi and K. Nate, Tetrahedron, 1971, 27, 2705-2711.

32 G. Torosian, H. McVeigh, P. J. Kovi and S. G. Schulman, Spectrosc. Lett., 1973, 6, 77-85.

33 M. Montalti, A. Credi, L. Prodi and M. T. Gandolfi, Handbook of Photochemistry, Taylor and Francis, Boca Raton, 3rd edn, 2006.

34 P. Debye, Trans. Electrochem. Soc., 1942, 82, 265-272.

35 M. Goez, Z. Phys.-Chem. Materialforsch., 1990, 169, 133-145.

36 G. Grabner, K. Rechthaler, B. Mayer, G. Köhler and K. Rotkiewicz, J. Phys. Chem. A, 2000, 104, 1365-1376.

37 C. L. Thomsen, D. Madsen, S. R. Keiding, J. Thgersen and O. Christiansen, J. Chem. Phys., 1999, 110, 3453-3462.

38 P. M. Hare, E. A. Price and D. M. Bartels, J. Phys. Chem. A, 2008, 112, 6800-6802.

39 H. Mauser, Z. Naturforsch., 1968, 23B, 1025-1030.

40 F.-M. Pang, C.-E. Seng, T.-T. Teng and M. H. Ibrahim, J. Mol. Liq., 2006, 136, 71-78.

41 U. Lachish, A. Shafferman and G. Stein, J. Chem. Phys., 1976, 64, 4205-4211.

42 M. B. Davies, J. Austin and D. A. Partridge, Vitamin C: Its Chemistry and Biochemistry, The Royal Society of Chemistry, Cambridge, 1st edn, 1991.

43 P. Wardman, J. Phys. Chem. Ref. Data, 1989, 18, 1637-1755. 